\title{
Elevated serum miR-133a predicts patients at risk of periprocedural myocardial injury after elective percutaneous coronary intervention
}

\author{
You Zhou*, Zhangwei Chen*, Ao Chen, Jiaqi Ma, Juying Qian, Junbo Ge \\ Department of Cardiology, Zhongshan Hospital, Fudan University, \\ Shanghai Institute of Cardiovascular Diseases, Shanghai, China
}

\begin{abstract}
Background: Periprocedural myocardial injury (PMI) is a frequent complication of percutaneous coronary intervention (PCI) associated with poor prognosis. However, no effective method has been found to identify patients at risk of PMI before the procedure. MicroRNA-133a (miR-133a) has been reported as a novel biomarker in various cardiovascular diseases. Herein, it was sought to determine whether circulating miR-133a could predict PMI before the procedure.

Methods: Eighty patients with negative preoperative values of cardiac troponin $T$ (cTnT) receiving elective PCI for stable coronary artery disease (CAD) were recruited. Venous serum samples were collected on admission and within 16-24 hours post-PCI for miRNA measurements. PMI was defined as a cTnT value above the 99\% upper reference limit after the procedure. The association between miR-133a and PMI was further assessed.

Results: Periprocedural myocardial injury occurred in 48 patients. The circulating level of miR-133a was significantly higher in patients with PMI before and after the procedure (both $p<0.001$ ). Receiver operating characteristic curve analysis of the preoperative miR-133a level revealed an area under the curve of 0.891, with a sensitivity of $93.8 \%$ and a specificity of $71.9 \%$ to predict PMI. Additionally, a decrease was found in fibroblast growth factor receptor 1 (FGFR1) in parallel with an increase in miR-133a levels in patients with PMI.

Conclusions: This study demonstrates for the first time that serum miR-133a can be used as a novel biomarker for early identification of stable CAD patients at risk of PMI undergoing elective PCI. The miR-133a-FGFR1 axis may be involved in the pathogenesis of PMI. (Cardiol J 2022; 29, 2: 284-292)

Key words: periprocedural myocardial injury, percutaneous coronary intervention, microRNA-133a
\end{abstract}

\section{Introduction}

With emerging innovations in techniques and equipment, percutaneous coronary intervention (PCI) has been dramatically improved and is one of the most commonly used approaches for coronary revascularization. Complications of PCI, such as acute stent thrombosis, coronary perforation, stroke and death, are critical but rarely occur. In contrast, periprocedural myocardial injury (PMI) is a frequent complication associated with a poor prognosis, which can be detected in $5-40 \%$ of cases, even after an uneventful PCI procedure [1-3]. Some risk factors for PMI, such as older age and renal dysfunction, can be identified before the procedure $[4,5]$. However, information about other

Address for correspondence: Dr. Juying Qian, MD, PhD, FESC, FACC or Junbo Ge, MD, PhD, FESC, FACC, FSCAI, Department of Cardiology, Zhongshan Hospital, Fudan University, Shanghai Institute of Cardiovascular Diseases, 180 Fenglin Road, Shanghai 200032, China, tel: 86-21-60268565 or tel: 86-21-64041990 ext. 2745, fax: 86-21-64223006, e-mail: qian.juying@zs-hospital.sh.cn or e-mail: jbge@zs-hospital.sh.cn

Received: 24.11.2019 Accepted: 26.02.2020 Early publication date: 18.03.2020

*You Zhou and Zhangwei Chen contributed equally to this article.

This article is available in open access under Creative Common Attribution-Non-Commercial-No Derivatives 4.0 International (CC BY-NC-ND 4.0) license, allowing to download articles and share them with others as long as they credit the authors and the publisher, but without permission to change them in any way or use them commercially. 
crucial predictors, such as multivessel disease, stent length and stent number, are not available until the procedure is finished. No method has been found to effectively identify patients at a high risk of PMI preoperatively.

MicroRNAs (miRNAs) represent a class of small noncoding RNAs that play an important role in posttranscriptional gene regulation by promoting mRNA degradation or direct translation inhibition. In the heart, miRNAs have been proposed as biomarkers in various cardiovascular diseases, such as heart failure [6], myocardial infarction [7] and arrhythmia [8]. miR-133a is a muscle-enriched miRNA that is critically involved in angiogenesis, apoptosis, cardiac fibrosis and hypertrophy [9]. In recent years, circulating miR-133a has been broadly reported as a biomarker of myocardial injury that correlates with troponin levels [9-12]. An elevated level of serum miR-133a in ST-elevation myocardial infarction patients is associated with more pronounced myocardial injury and a higher incidence of major adverse cardiovascular events [13]. However, whether the miR-133a signature is altered in stable coronary artery disease (CAD) patients with PMI has not been thoroughly investigated.

The present study was designed to ascertain the circulating level of miR-133a both before and after PCI. Furthermore, it was sought to determine whether PMI is associated with changes in miR-133a expression that could be identified preoperatively so that miR-133a may be used as a novel biomarker to predict PMI and rationalize early preventive strategies.

\section{Methods}

\section{Ethics statement}

This study was approved by the Ethical Committee of Zhongshan Hospital, Fudan University (Approval NO: B2016-018, Date: 2016/02/29), and all patients gave their written, informed consent. The study was conducted in accordance with the tenets of the Declaration of Helsinki.

\section{Patient population}

Eighty stable CAD patients with negative preoperative cardiac troponin T (cTnT) levels scheduled for elective PCI were enrolled in Zhongshan Hospital, Fudan University. Patients with unstable angina pectoris, acute myocardial infarction, New York Heart Association class III-IV heart failure, moderate-to-severe valvular disease or bypass surgery history were excluded. Other exclusion crite- ria were active infection, respiratory failure, chronic kidney disease (eGFR $<60 \mathrm{~mL} / \mathrm{min} / 1.73 \mathrm{~m}^{2}$ ), liver dysfunction (liver enzyme $>3 \times$ upper reference limit [URL] or bilirubin $>2 \times$ URL), malignancy and systemic connective tissue disease. Patients with a cTnT level $>0.03 \mathrm{ng} / \mathrm{mL}(99 \%$ URL) within 16-24 hours post-PCI were assigned to the PMI group.

\section{Serum collection and storage}

Venous blood samples were taken on admission before angiography and $16-24$ hours post-PCI. The serum was separated by centrifugation at $1000 \mathrm{~g}$ for $10 \mathrm{~min}$ at $4^{\circ} \mathrm{C}$. The supernatant was carefully transferred to RNase/DNase-free cryovials and stored at $-80^{\circ} \mathrm{C}$ until assayed.

\section{Measurement of serum miRNA}

Total RNA was isolated from $800 \mu \mathrm{L}$ of serum and reverse transcribed according to the manufacturer's instructions (GenePharma, Shanghai). Circulating levels of miRNAs were validated by quantitative real-time polymerase chain reaction (PCR) performed with specific TaqMan miRNA assays (Cat. " GMRS-001, GenePharma, Shanghai) using MX3000P (Stratagene, US). miR-16 was used as an internal standard to normalize the miRNA content, expressed as $2^{-\mathrm{CT}(\mathrm{miR}-16)}$, and normalized to the mean value of the plate. The relative quantitation of $\mathrm{miR}-133 \mathrm{a}$ is expressed as $2^{-\mathrm{CT}(\mathrm{miRNA})} /$ normalized miR-16 values, i.e., $2^{-\Delta \mathrm{CT}}$. The miRNA primers used in the assay are shown in Table 1.

\section{Serum fibroblast growth factor receptor 1 (FGFR1) detection}

The serum level of FGFR1 was determined in $100 \mu \mathrm{L}$ of serum using a quantitative sandwich enzyme immunoassay (Cat. ${ }^{*}$ PEL-FGFR1- $\gamma$, RayBiotech, Norcross, GA) in accordance with the manufacturer's instructions.

\section{Statistical analyses}

Continuous variables are presented as mean \pm standard deviation (SD) or as median with the interquartile range (IQR). Categorical data are expressed as counts and percentages. Differences between groups were assessed using the Fisher exact test or the $\chi^{2}$ test for categorical variables. The Student $t$ test and the Mann-Whitney U test were used to assess differences between groups in normally and non-normally distributed continuous variables, respectively. Univariable binary logistic regression analyses were performed to identify 
Table 1. MicroRNA primer sequences.

\begin{tabular}{lcc}
\hline MicroRNA & Sequence & \\
\hline \multirow{3}{*}{ hsa-mir-16 } & F primer & AAGCACCTAGCAGCACGTAAATA \\
& R primer & TATGGTTTTGACGACTGTGTGAT \\
Size: $67 \mathrm{bp}$ & \\
hsa-mir-133a & F primer & GCCTTTGGTCCCCTTCAAC \\
& R primer & TATGCTTGTTCTCGTCTCTGTGTC \\
\hline
\end{tabular}

Table 2. Baseline demographics and clinical characteristics.

\begin{tabular}{|c|c|c|c|c|}
\hline & Total $(\mathbf{n}=80)$ & Non-PMI (n = 32) & PMI (n = 48) & $\mathbf{P}$ \\
\hline Age & $63.1 \pm 9.8$ & $62.1 \pm 10.6$ & $64.5 \pm 9.8$ & 0.358 \\
\hline Male & $55(68.8 \%)$ & $22(68.8 \%)$ & $33(68.8 \%)$ & 1.000 \\
\hline Hypertension & $48(60.0 \%)$ & $20(62.5 \%)$ & $28(58.3 \%)$ & 0.709 \\
\hline Diabetes & $21(26.3 \%)$ & $6(18.8 \%)$ & $15(31.3 \%)$ & 0.213 \\
\hline Smoking history & $28(35.0 \%)$ & $11(34.4 \%)$ & $17(35.4 \%)$ & 0.924 \\
\hline Prior $\mathrm{PCl}$ & $18(22.5 \%)$ & $5(15.6 \%)$ & $13(27.1 \%)$ & 0.229 \\
\hline \multicolumn{5}{|c|}{ Laboratory and auxiliary examinations } \\
\hline Creatinine [mg/dL] & $77.5 \pm 16.6$ & $77.7 \pm 16.6$ & $77.4 \pm 16.8$ & 0.951 \\
\hline CK [U/L] & $99.6 \pm 53.6$ & $114.5 \pm 67.9$ & $90.0 \pm 39.8$ & 0.228 \\
\hline CK-MB [U/L] & $16.6 \pm 7.4$ & $16.5 \pm 10.4$ & $16.7 \pm 4.7$ & 0.195 \\
\hline hs-CRP [mg/L] & $2.4 \pm 4.3$ & $1.5 \pm 1.7$ & $3.0 \pm 5.4$ & 0.502 \\
\hline White blood cell count $\left[\times 10^{9} / \mathrm{L}\right]$ & $6.24 \pm 1.63$ & $5.96 \pm 1.71$ & $6.42 \pm 1.57$ & 0.230 \\
\hline Platelet $\left[\times 10^{9} / \mathrm{L}\right]$ & $208.2 \pm 55.6$ & $207.1 \pm 41.3$ & $209.0 \pm 63.7$ & 0.818 \\
\hline Total cholesterol [mmol/L] & $3.72 \pm 0.91$ & $3.54 \pm 0.70$ & $3,83 \pm 1.01$ & 0.260 \\
\hline LDL [mmol/L] & $1.84 \pm 0.79$ & $1.72 \pm 0.53$ & $1.91 \pm 0.92$ & 0.584 \\
\hline Triglycerides [mmol/L] & $1.84 \pm 1.15$ & $2.02 \pm 1.45$ & $1.73 \pm 0.91$ & 0.617 \\
\hline $\mathrm{HDL}[\mathrm{mmol} / \mathrm{L}]$ & $1.11 \pm 0.33$ & $1.07 \pm 0.31$ & $1.14 \pm 0.34$ & 0.393 \\
\hline Lp(a) [mmol/L] & $287.0 \pm 339.6$ & $229.1 \pm 238.1$ & $324.7 \pm 389.8$ & 0.407 \\
\hline HbA1c [\%] & $6.05 \pm 0.80$ & $6.08 \pm 0.82$ & $6.04 \pm 0.79$ & 0.823 \\
\hline \multicolumn{5}{|l|}{ Echocardiography } \\
\hline Left atrium [mm] & $38.5 \pm 3.8$ & $39.7 \pm 4.3$ & $38.2 \pm 3.4$ & 0.475 \\
\hline LVEDD [mm] & $46.1 \pm 3.9$ & $46.8 \pm 3.9$ & $45.7 \pm 3.9$ & 0.210 \\
\hline SPAP $[\mathrm{mmHg}]$ & $32.5 \pm 7.2$ & $33.0 \pm 7.4$ & $32.1 \pm 7.1$ & 0.184 \\
\hline Ejection fraction [\%] & $64.6 \pm 8.1$ & $65.6 \pm 2.9$ & $63.9 \pm 10.1$ & 0.365 \\
\hline
\end{tabular}

Data are shown as mean \pm standard deviation or number (\%). CK — creatine kinase; hs-CRP — high sensitive C-reactive protein; HDL — high density lipoprotein; LDL — low density lipoprotein; LVEDD — left ventricular end-diastolic diameter; Lp(a), lipoprotein (a); PMI — periprocedural myocardial injury; $\mathrm{PCl}$ — percutaneous coronary intervention; SPAP — systolic pulmonary arterial pressure

the risk factors for PMI. Correlation analyses were performed using the Spearman tests. A receiver operating characteristic (ROC) curve was established to predict patients with PMI. All probability values were two-tailed, and $\mathrm{p}<0.05$ was considered significant. All analyses were performed with SPSS for Windows, release 25.0 (IBM SPSS, Inc., Chicago, IL, USA). Figures were plotted using
GraphPad Prism, version 6.0 (GraphPad Software, San Diego, CA, USA).

\section{Results}

\section{Baseline and procedural characteristics}

The baseline data for the recruited patients are summarized in Table 2 . Briefly, most patients 
Table 3. Angiographic and procedural features.

\begin{tabular}{|c|c|c|c|c|}
\hline & Total $(n=80)$ & Non-PMI (n = 32) & PMI (n = 48) & $\mathbf{P}$ \\
\hline Syntax score & $14.1 \pm 8.5$ & $10.4 \pm 5.2$ & $16.6 \pm 9.4$ & 0.002 \\
\hline СТО & $8(10.0 \%)$ & $2(6.3 \%)$ & $6(12.5 \%)$ & 0.466 \\
\hline \multicolumn{5}{|l|}{ Target vessel number } \\
\hline 1 & $57(71.3 \%)$ & $30(93.8 \%)$ & $27(56.3 \%)$ & $<0.001$ \\
\hline 2 & $20(25.0 \%)$ & $2(6.2 \%)$ & $18(37.4 \%)$ & $<0.001$ \\
\hline 3 & $3(3.8 \%)$ & - & $3(6.3 \%)$ & $<0.001$ \\
\hline Multivessel disease* & $23(28.8 \%)$ & $2(6.2 \%)$ & $21(43.7 \%)$ & $<0.001$ \\
\hline Rotablation & $2(2.5 \%)$ & $1(3.1 \%)$ & $1(2.1 \%)$ & 1 \\
\hline PTCA without stenting & $1(1.2 \%)$ & $1(3.1 \%)$ & - & $<0.001$ \\
\hline \multicolumn{5}{|l|}{ Stent number } \\
\hline 1 & $36(45.0 \%)$ & $21(65.6 \%)$ & $15(31.3 \%)$ & 0.001 \\
\hline 2 & $21(26.2 \%)$ & $10(31.3 \%)$ & $11(22.9 \%)$ & 0.407 \\
\hline 3 & $15(18.8 \%)$ & - & $15(31.3 \%)$ & $<0.001$ \\
\hline 4 & $7(8.8 \%)$ & - & $7(14.6 \%)$ & $<0.001$ \\
\hline Stent length [mm] & $54.2 \pm 33.0$ & $35.5 \pm 15.3$ & $66.6 \pm 35.8$ & $<0.001$ \\
\hline
\end{tabular}

Data are shown as mean \pm standard deviation or number (\%). ${ }^{*}$ Means target vessel number $\geq 2$; CTO — chronic total occlusion; PMI — periprocedural myocardial injury; PTCA — percutaneous transluminal coronary angioplasty

were men $(58.8 \%)$, with a mean age of 63.1 years. The level of cTnT was elevated above the $99 \%$ upper reference limit (URL) in 48 patients after the procedure. Traditional risk factors for CAD, such as hypertension, diabetes, smoking history and dyslipidemia, were not significantly different in patients with or without PMI. Procedural features are presented in Table 3. Patients with PMI had a higher Syntax score $(16.6 \pm 9.4$ vs. $10.4 \pm 5.2$, $\mathrm{p}=0.002$ ), indicating more complex coronary lesions. Compared with patients without PMI, more stents were implanted into patients with PMI, whose total stent length, as a result, was longer $(66.6 \pm 35.8$ vs. $35.5 \pm 15.3 \mathrm{~mm}, \mathrm{p}<0.001)$. In addition, multivessel disease (defined as target vessels $\geq 2$ ) was more common in patients with PMI $(6.2 \%$ vs. $43.8 \%, \mathrm{p}<0.001)$. Echocardiography revealed no difference in the ejection fraction (EF), left ventricular end-diastolic diameter (LVEDD), left atrial diameter or systolic pulmonary arterial pressure.

\section{Circulating miRNA profiles and $\mathrm{cTn} T$ values}

As shown in Figure 1, the baseline cTnT values were similar between groups. The median concentration of cTnT after PCI was $0.109 \mathrm{ng} / \mathrm{mL}$ (IQR 0.050-0.220) in the PMI group, corresponding to $0.016 \mathrm{ng} / \mathrm{mL}$ (IQR $0.011-0.021$ ) in the non-PMI group. Of importance, the serum level of miR-133a was significantly increased in patients with PMI, even before the procedure $(\mathrm{p}<0.001)$. In addition, the circulating signature of miR-133a did not change in either group after revascularization, that is, miR-133a was still substantially elevated in patients with PMI after PCI. The correlation between miR-133a and cTnT was positive based on the Spearman test $\left(r_{s}=0.281, p<0.001\right)$, which, however, was not significantly high.

\section{Predictive value of circulating miR-133a for PMI}

Binary logistic regression analyses demonstrated that traditional risk factors for coronary heart disease, such as older age, hypertension, diabetes, and smoking history, did not predispose patients to PMI (Table 4). In contrast, stent length $(\geq 30 \mathrm{~mm})$, stent number as well as multivessel disease were associated with a higher risk of PMI, which, as mentioned above, could not be determined before the procedure. Of importance, ROC curve analyses implied that the preoperative miR-133a level could accurately predict PMI, with a sensitivity of $93.8 \%$, a specificity of $71.9 \%$ and an area under the curve (AUC) of 0.891 (95\% confidence interval [CI] 0.818-0.965) (Fig. 2). Furthermore, demonstrated herein, was that high circulating miR-133a $\left(2^{-\Delta \mathrm{CT}}>0.04427\right.$, the cut-off value derived from ROC analyses) increased the risk of PMI. After adjustment for stent length 


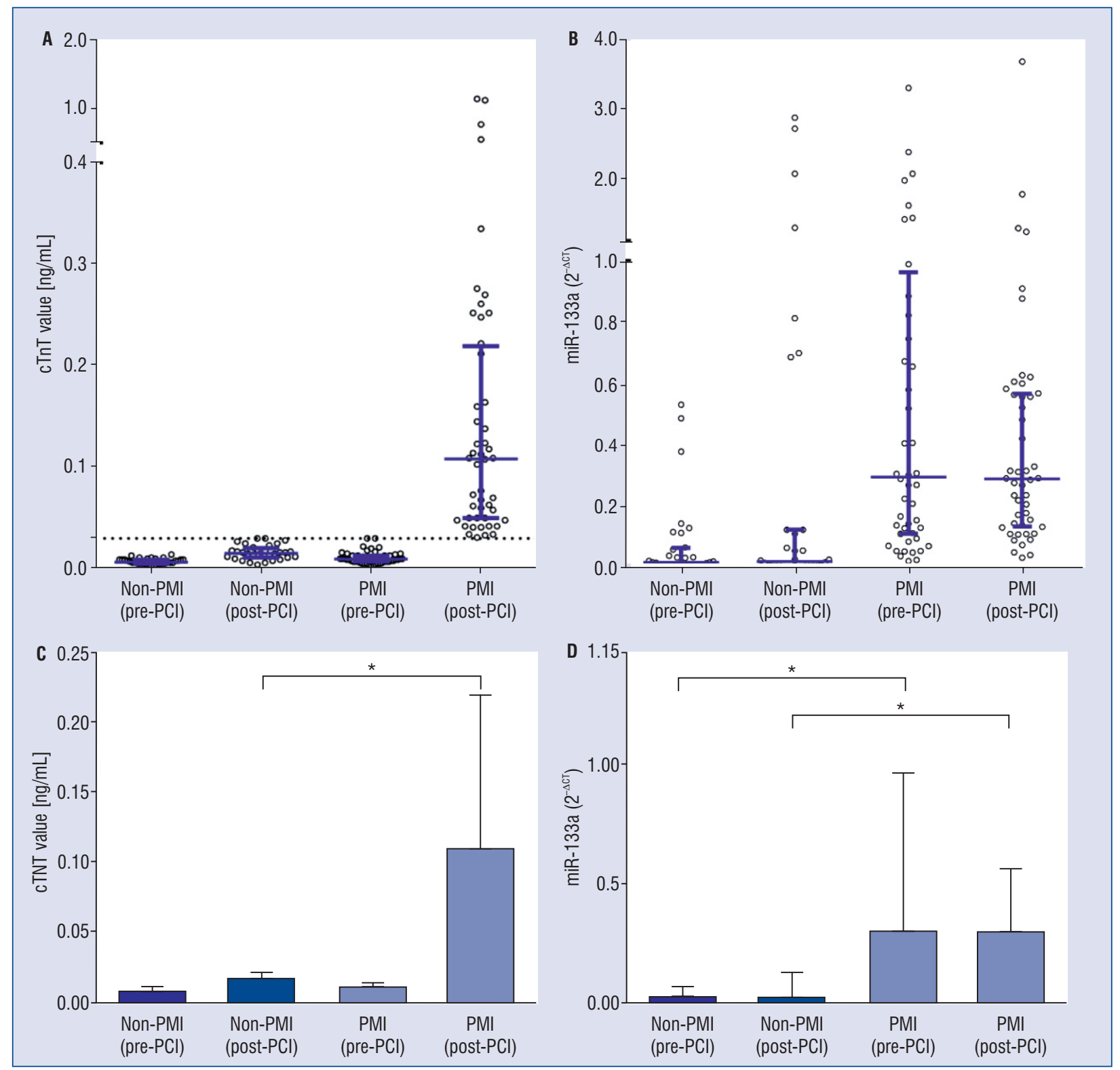

Figure 1. Periprocedural serum levels of miR-133a and cardiac troponin $T(c T n T) ; A$, B. Distribution of serum levels of miR-133a and cTnT in patients with or without periprocedural myocardial injury (PMI). Median and interquartile range of each category are shown in blue lines. Dotted line represents the $99 \%$ upper reference limit of cTnT; C, D. Comparison of miR-133a and cTnT levels. MiR-133a was substantially elevated in patients with PMI both before and after the procedure; ${ }^{*} p<0.001$.

( $\geq 30 \mathrm{~mm}$ ), stent number and multivessel disease, it was found that elevated preoperative serum miR-133a was an independent risk factor of PMI (adjusted odds ratio $=34.20,95 \%$ CI 6.34-184.39, data not shown).

\section{miR-133a and FGFR1 expression}

The FGFR1, a previously proven target of miR-133a, has been reported to be critically in- volved in glucose and lipid metabolism, oxidative stress and inflammatory responses, which seems to be associated with the pathogenesis of PMI. Therefore, we measured the serum level of FGFR1 and discovered that FGFR1 was significantly decreased in patients with PMI in parallel with the upregulation of miR-133a (Fig. 3), suggesting a potential role of the miR-133a-FGFR1 axis in the pathogenesis of PMI. 
Table 4. Risk factors of periprocedural myocardial injury.

\begin{tabular}{lcc}
\hline & Odds ratio $(95 \% \mathrm{CI})$ & $\mathrm{P}$ \\
\hline Age $\geq 65$ years & $2.05(0.82-5.08)$ & 0.123 \\
Male & $1.00(0.38-2.63)$ & 1 \\
Hypertension & $0.84(0.34-2.10)$ & 0.709 \\
Diabetes & $1.97(0.67-5.78)$ & 0.217 \\
Smoking history & $1.05(0.41-2.68)$ & 0.924 \\
Prior PCI & $2.01(0.64-6.32)$ & 0.234 \\
hs-CRP & $1.13(0.95-1.34)$ & 0.167 \\
LDL cholesterol & $1.39(0.73-2.65)$ & 0.322 \\
Stent number & $4.06(1.99-8.31)$ & $<0.001$ \\
Stent length $(\geq 30 \mathrm{~mm})$ & $3.00(1.06-8.52)$ & 0.039 \\
Multivessel disease* & $11.7(2.50-54.46)$ & 0.002 \\
miR-133a $\left(2^{-\Delta C T}>0.04427\right)^{* *}$ & $38.33(9.45-155.43)$ & $<0.001$ \\
\hline
\end{tabular}

*Means target vessel number $\geq 2$. **The cut-off value was derived from receiver operating characteristic analyses shown in Figure 2 . Due to relatively small sample umber, the $95 \%$ confidence international (Cl) was wide. LDL — low-density lipoprotein; hs-CRP — high sensitive C-reactive protein; $\mathrm{PCl}$ — percutaneous coronary intervention

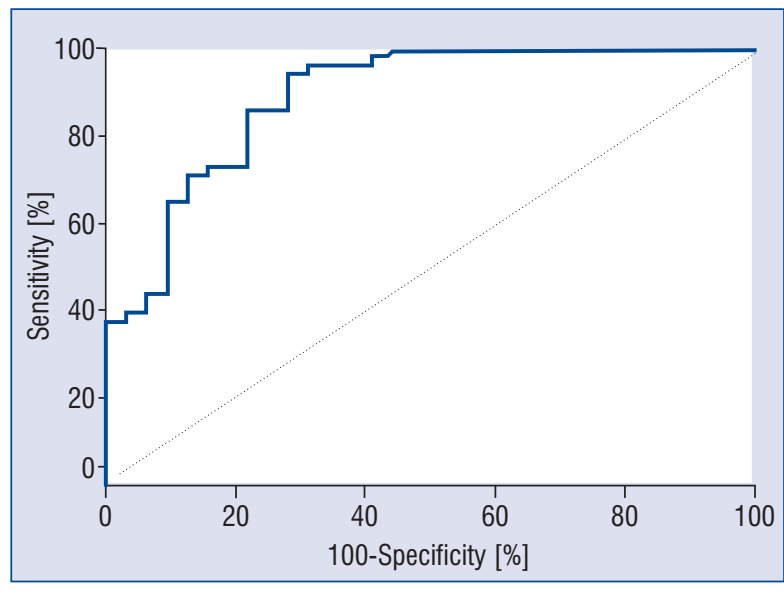

Figure 2. Receiver operating characteristic curve for the prediction of periprocedural myocardial injury (PMI). The dotted diagonal line is the Null Hypothesis with area under the curve (AUC) $=0.500$. MiR-133a $\left(2^{-\triangle C T}\right.$ $>0.04427$ ) had a sensitivity of $93.8 \%$ and specificity of $71.9 \%$ to predict PMI with AUC $=0.891$ (95\% confidence interval 0.818-0.965).

\section{Discussion}

In the present study, we evaluated the expression profile of circulating miR-133a in patients with and without PMI. Quantitative RT-PCR assessment implied that the serum level of miR-133a was significantly higher in patients with PMI both before and after the procedure. The present data demonstrated for first time that miR-133a was an independent risk

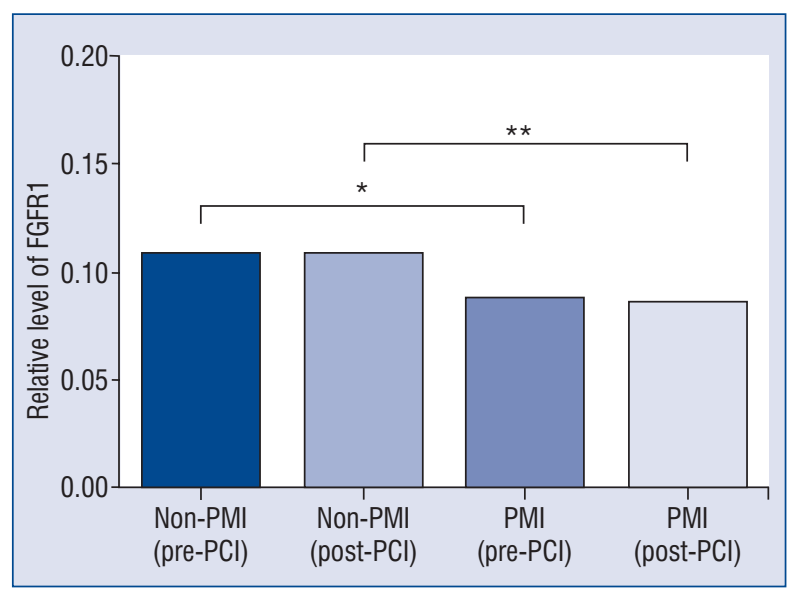

Figure 3. Serum level of fibroblast growth factor receptor 1 (FGFR1). Circulating levels of FGFR1. FGFR1 was significantly down-regulated in patients with periprocedural myocardial injury (PMI) both before and after the procedure; ${ }^{*} p<0.05 ;{ }^{*} p<0.01$.

factor of PMI and preoperative value of miR-133a could be used to accurately predict PMI in stable CAD patients undergoing elective PCI. As a target of miR-133a, the serum level of FGFR1 was correspondingly downregulated in patients with PMI, indicating that the miR-133a-FGFR1 axis may be involved in the pathological mechanism of PMI.

No effective method has been validated to distinguish patients at an increased risk of PMI 
preoperatively. Some of the key factors that influence the incidence and magnitude of PMI could be discerned before the procedure, such as older age, pre-existing renal impairment, and the presence of anemia [4, 14]. Nevertheless, angiographic and procedure-related factors, such as plaque burden, bifurcation lesions, stent number, stent length, the use of a rotablator or directional coronary atherectomy, cannot be ascertained until the procedure is finished [4]. Cardiac troponin can detect PMI only when risk factors take their toll. The delayed identification of PMI hinders the early adoption of a preventive strategy.

It is acknowledged that PMI is closely related with two mechanisms: side branch loss and microcirculatory obstruction (structurally or functionally) [3]. Complex lesions and procedures were crucial determinants of PMI, which were associated with higher risk of side branch loss and microembolization from plaque debris. However, complex lesions or procedures are not bound to cause PMI, and uneventful PCI could lead to PMI. Since, presently it was believed complex lesions and procedures are components, but not the sole determinant of PMI. In this study, side branch obstruction was not found after double-checking each angiogram, which indicated the dominant role of coronary microcirculatory dysfunction. It was hypothesized herein, that disrupted microcirculation before PCI, disposed patients to a higher incidence of PMI and adverse cardiovascular events.

Owing to their high stability in different biofluids, including serum, plasma and urine [15], the potential for miRNAs to serve as noninvasive biomarkers has drawn great interest. miR-133a is a muscle-enriched miRNA that has been reported to be increased in patients with myocardial infarction and is closely related to cardiac troponin [16]. Gacoń et al. [17] observed that miR-133a were significantly elevated in myocardial infraction patients with an occluded culprit coronary artery compared to those with patent culprit artery, while troponin levels did not differ between the two groups. This discordance between miRNA and cTn was also found in the present study, which implied higher sensitivity of miR-133a or a more complex role miR-133a played in myocardial injury. Numerous studies confirmed that miR-133a was deeply involved in the pathological mechanisms of endothelial dysfunction [18], vascular smooth muscle cells proliferation and differentiation [19], as well as myocardial inflammation [20], which all could compromise the microvascular system. It was presently speculated that miR-133a may not only be a reflection of myocardial damage, but also a crucial risk factor to precipitate PMI. Notably, it was demonstrated that microvascular dysfunction reflected by index of microcirculatory resistance (IMR) was positively correlated with cTn values, and patients developed type 4a myocardial infarction had significantly higher IMR levels both at baseline and post-PCI [21]. Besides, IMR were significantly increased after PCI in patients who developed type 4a myocardial infarction compared with those who did not. Consequently, it was believed that microcirculatory dysfunction did predispose patients to PMI, and PMI could invertedly deteriorate microcirculatory dysfunction. Unfortunately, IMR measurement couldn't be accomplished in the present center to correlate IMR with miR-133a. Nevertheless, considering the current findings and previously published results, it was believed it would be convincing that a high level of serum miR-133a served as an index of microvascular dysfunction which disposed patients to high risk of PMI. The serum level of miR-133a was measured both before and after the procedure. Circulating miR-133a was significantly upregulated in PMI patients before PCI was performed. ROC analyses indicated that miR-133a could be used as a novel biomarker to distinguish patients at high risk of PMI, with an AUC of 0.891. Using the cut-off value derived from ROC analyses, a high level of preoperative circulating miR-133a was demonstrated and was an independent risk factor of PMI. Partially due to the relatively small sample number, the $95 \% \mathrm{CI}$ of odds ratio was wide and other baseline predictors, including age, diabetes, and prior PCI, did not increase the risk of PMI in this study. In addition, although the correlation of miR-133a and cTnT was positive, consistent with previous reports, the coefficient was not high. Different temporal releasing patterns may be one of the possible explanations for this inconsistency. Large-scale research is needed to validate the results and integrate multiple variables to generate a more precise model to predict PMI.

This study provides the first documented insights into the miR-133a signature of PMI. The increased level of miR-133a before PCI implied a potential initiating role in the pathogenesis of PMI. FGFR1, as a target of miR-133a, is an important regulator in lipid and glucose metabolism [22], obesity, myocardial development, cardiac functional recovery [23], as well as vascular development and inflammation [24], which could possibly affect microcirculation. Hence, the serum level of FGFR1 was subsequently measured. In parallel with the 
increase in miR-133a, FGFR1 was significantly downregulated in patients with PMI both before and after the procedure. Therefore, a potential role of the miR-133a-FGFR1 axis in the pathogenesis of PMI can be speculated. The specific mechanism revealed herein was preliminary. More specific mechanisms of the miR-133a axis deserves further research.

\section{Limitations of the study}

There are some limitations acknowledged in the present study. First, the sample size was relatively small, and subjects were confined to stable CAD patients scheduled for elective PMI. Patients with active infection, respiratory failure, chronic kidney disease and so on were excluded in this study because these complications would influence the level of cTnT (in other words, PMI) independent of the interventional procedure. Large-scale research is required to confirm the results in broader populations. Second, only one serum sample was collected before and after PCI. More serum samples at different times are required to determine the time course of circulating miR-133a. Third, the exact source of the increased levels of miR-133a was not ascertained because miR-133a is also enriched in skeletal muscle. However, considering that creatine kinase values are not significantly different in patients with and without PMI, miR-133a is most likely released from the myocardium. Fourth, the pathogenesis of PMI is a multifactorial process, and the miR-133a-FGFR1 axis is unlikely to be the sole underlying mechanism. Finally, the clinical application of miR-133a still relies on the development of robust and simple detection methods and validation of the cut-off values.

\section{Conclusions}

For the first time, this study demonstrated the overexpression of circulating miR-133a in patients with PMI both before and after PCI. miR-133a was an independent risk factor of PMI. The preoperative miR-133a level can be used as a novel biomarker to accurately predict PMI in patients with stable CAD undergoing elective PCI. In addition, miR-133a-FGFR1 may be involved in the pathological mechanism of PMI.

\section{Acknowledgments}

This study was supported by National Natural Science Foundation of China: Grant No. 81970295 , 81870267, 81670318 and 81570314, Grant of Shanghai Municipal Commission of Health and
Family Planning: Grant No. 2017YQ057, Grant of Shanghai Science and Technology Committee: Grant No. 17411962300, the National Program on Key Basic Research Project of China: 973 Program, Grant No. 2014CBA02003, Grant of Zhongshan Hospital Affiliated to Fudan University: Grant No. 2015ZSYXGG07, 2017ZSYQ08 and Zhupei-10, VG Funding of Clinical Trials: 2017-CCA-VG-036, and Merck Funding: Xinxin-merck-fund-051, Program for Outstanding Medical Academic Leader: Grant No. 2015-Weijiwei-24.

\section{Conflict of interest: None declared}

\section{References}

1. Califf RM, Abdelmeguid AE, Kuntz RE, et al. Myonecrosis after revascularization procedures. J Am Coll Cardiol. 1998; 31(2): 241-251, doi: 10.1016/s0735-1097(97)00506-8, indexed in Pubmed: 9462562.

2. Prasad A, Rihal CS, Lennon RJ, et al. Significance of periprocedural myonecrosis on outcomes after percutaneous coronary intervention: an analysis of preintervention and postintervention troponin T levels in 5487 patients. Circ Cardiovasc Interv. 2008; 1(1): 10-19, doi: 10.1161/CIRCINTERVENTIONS.108.765610, indexed in Pubmed: 20031650.

3. Babu GG, Walker JM, Yellon DM, et al. Peri-procedural myocardial injury during percutaneous coronary intervention: an important target for cardioprotection. Eur Heart J. 2011; 32(1): 23-31, doi: 10.1093/eurheartj/ehq393, indexed in Pubmed: 21037252.

4. Park DW, Kim YH, Yun SC, et al. Frequency, causes, predictors, and clinical significance of peri-procedural myocardial infarction following percutaneous coronary intervention. Eur Heart J. 2013; 34(22): 1662-1669, doi: 10.1093/eurheartj/eht048, indexed in Pubmed: 23404537.

5. Zeitouni M, Silvain J, Guedeney P, et al. ACTION Study Group. Periprocedural myocardial infarction and injury in elective coronary stenting. Eur Heart J. 2018; 39(13): 1100-1109, doi: 10.1093/eurheartj/ehx799, indexed in Pubmed: 29365133.

6. Kalozoumi G, Yacoub M, Sanoudou D. MicroRNAs in heart failure: Small molecules with major impact. Glob Cardiol Sci Pract. 2014; 2014(2): 79-102, doi: 10.5339/gcsp.2014.30, indexed in Pubmed: 25419522.

7. Navickas R, Gal D, Laucevičius A, et al. Identifying circulating microRNAs as biomarkers of cardiovascular disease: a systematic review. Cardiovasc Res. 2016; 111(4): 322-337, doi: 10.1093/ cvr/cvw174, indexed in Pubmed: 27357636.

8. Harling L, Lambert J, Ashrafian H, et al. Elevated serum microRNA 483-5p levels may predict patients at risk of post-operative atrial fibrillation. Eur J Cardiothorac Surg. 2017; 51(1): 73-78, doi: 10.1093/ejcts/ezw245, indexed in Pubmed: 27422887.

9. Xiao Yi, Zhao J, Tuazon JP, et al. MicroRNA-133a and myocardial infarction. Cell Transplant. 2019; 28(7): 831-838, doi: 10.1177/0963689719843806, indexed in Pubmed: 30983393.

10. Schulte C, Barwari T, Joshi A, et al. Comparative analysis of circulating noncoding rnas versus protein biomarkers in the detection of myocardial injury. Circ Res. 2019; 125(3): 328-340, doi: 10.1161/CIRCRESAHA.119.314937, indexed in Pubmed: 31159652. 
11. Jaguszewski M, Osipova J, Ghadri JR, et al. A signature of circulating microRNAs differentiates takotsubo cardiomyopathy from acute myocardial infarction. Eur Heart J. 2014; 35(15): 999-1006, doi: 10.1093/eurheartj/eht392, indexed in Pubmed: 24046434.

12. De Rosa S, Fichtlscherer S, Lehmann R, et al. Transcoronary concentration gradients of circulating microRNAs. Circulation. 2011; 124(18): 1936-1944, doi: 10.1161/CIRCULATIONAHA.111.037572, indexed in Pubmed: 21969012.

13. Eitel I, Adams V, Dieterich P, et al. Relation of circulating MicroRNA-133a concentrations with myocardial damage and clinical prognosis in ST-elevation myocardial infarction. Am Heart J. 2012; 164(5): 706-714, doi: 10.1016/j.ahj.2012.08.004, indexed in Pubmed: 23137501.

14. McKechnie RS, Smith D, Montoye C, et al. Prognostic implication of anemia on in-hospital outcomes after percutaneous coronary intervention. Circulation. 2004; 110(3): 271-277, doi: 10.1161/01. CIR.0000134964.01697.C7, indexed in Pubmed: 15226214.

15. Xu J, Zhao J, Evan G, et al. Circulating microRNAs: novel biomarkers for cardiovascular diseases. J Mol Med (Berl). 2012; 90(8): 865-875, doi: 10.1007/s00109-011-0840-5, indexed in Pubmed: 22159451.

16. D'Alessandra Y, Devanna P, Limana F, et al. Circulating microRNAs are new and sensitive biomarkers of myocardial infarction. Eur Heart J. 2010; 31(22): 2765-2773, doi: 10.1093/eurheartj/ ehq167, indexed in Pubmed: 20534597.

17. Gacoń J, Kabłak-Ziembicka A, Stępień E, et al. Decision-making microRNAs (miR-124, -133a/b, -34a and -134) in patients with occluded target vessel in acute coronary syndrome. Kardiol Pol. 2016; 74(3): 280-288, doi: 10.5603/KP.a2015.0174, indexed in Pubmed: 26365938.

18. Li P, Yin YL, Guo T, et al. Inhibition of Aberrant MicroRNA-133a Expression in Endothelial Cells by Statin Prevents Endothe- lial Dysfunction by Targeting GTP Cyclohydrolase 1 in Vivo. Circulation. 2016; 134(22): 1752-1765, doi: 10.1161/CIRCULATIONAHA.116.017949, indexed in Pubmed: 27765794.

19. Torella D, Iaconetti C, Catalucci D, et al. MicroRNA-133 controls vascular smooth muscle cell phenotypic switch in vitro and vascular remodeling in vivo. Circ Res. 2011; 109(8): 880-893, doi: 10.1161/CIRCRESAHA.111.240150, indexed in Pubmed: 21852550.

20. Rubiś P, Totoń-Żurańska J, Wiśniowska-Śmiałek S, et al. The relationship between myocardial fibrosis and myocardial microRNAs in dilated cardiomyopathy: A link between mir-133a and cardiovascular events. J Cell Mol Med. 2018; 22(4): 2514-2517, doi: 10.1111/jcmm.13535, indexed in Pubmed: 29377565.

21. Mangiacapra F, Bressi E, Di Gioia G, et al. Coronary microcirculation and peri-procedural myocardial injury during elective percutaneous coronary intervention. Int J Cardiol. 2020; 306: 42-46, doi: 10.1016/j.ijcard.2019.12.042, indexed in Pubmed: 31926642.

22. Foltz IN, Hu S, King C, et al. Treating diabetes and obesity with an FGF21-mimetic antibody activating the $\beta$ Klotho/FGFR1c receptor complex. Sci Transl Med. 2012; 4(162): 162-153, doi: 10.1126/ scitranslmed.3004690, indexed in Pubmed: 23197570.

23. Pennisi DJ, Mikawa T. FGFR-1 is required by epicardium-derived cells for myocardial invasion and correct coronary vascular lineage differentiation. Dev Biol. 2009; 328(1): 148-159 , doi: 10.1016/j.ydbio.2009.01.023, indexed in Pubmed: 19389363.

24. Wei W, Li XX, Xu M. Inhibition of vascular neointima hyperplasia by FGF21 associated with FGFR1/Syk/NLRP3 inflammasome pathway in diabetic mice. Atherosclerosis. 2019; 289: 132-142, doi: 10.1016/j.atherosclerosis.2019.08.017, indexed in Pubmed: 31513948. 